\title{
Body image dissatisfaction in patients with inflammatory bowel disease: a systematic review protocol
}

\author{
Sophie Elizabeth Beese ${ }^{*}$ (D), Isobel Marion Harris, David Moore and Janine Dretzke
}

\begin{abstract}
Background: Inflammatory bowel disease (IBD) is a debilitating chronic disease characterised by inflammation and ulceration of the gastrointestinal tract. It is associated with a range of debilitating symptoms and reduced quality of life. People living with IBD may also be at risk of body image dissatisfaction (BID). BID is a distorted and negative view of the physical self, which in turn can adversely affect mental health and quality of life. To date, there have been no systematic reviews of the evidence on BID in IBD patients. Therefore, the aim of this systematic review is to clarify the evidence base on BID in *IBD patients including (i) the tools used to measure $B I D$, (ii) the prevalence and severity of BID, (iii) the risk factors associated with BID and (iv) the relationship between BID and quality of life.

Methods: Bibliographic databases (EMBASE, MEDLINE, PsycINFO, Cochrane CENTRAL) will be searched using a sensitive search strategy aiming to identify any quantitative study reporting on body image in the context of IBD. This will be supplemented by searches of ongoing trials registers and checking of reference lists. Studies will be assessed for eligibility using predetermined selection criteria for each question. Data will be extracted using a predefined data extraction form, and risk of bias (quality) of included studies will be assessed based on checklists appropriate to the study designs identified. Key methodological steps will be undertaken in duplicate to minimise bias and error. Synthesis will be undertaken separately for the different systematic review sub-questions. Given the anticipated heterogeneity of evidence on each question, it is likely that synthesis will be mostly narrative.

Discussion: To the best of our knowledge, this will be the first systematic review to collate the existing evidence on BID in IBD patients. Understanding the impact of BID, its relationship with quality of life, and which patients may be at greater risk, may ultimately lead to the development of interventions to prevent or treat BID and to better patient care. Any gaps in the identified evidence will help to inform the research agenda in this area.

Systematic review registration: PROSPERO: (CRD42018060999).
\end{abstract}

Keywords: Systematic review, Inflammatory bowel disease, Ulcerative colitis, Crohn's disease, Body image

\section{Background}

Inflammatory bowel disease (IBD) is a chronic gastrointestinal disease characterised by inflammation and ulceration of the gastrointestinal tract. IBD consists of two main forms: Crohn's disease and ulcerative colitis [1]. Ulcerative colitis affects the large intestine, whereas Crohn's disease can affect the entire digestive tract [2]. Around 300,000 people in the UK are affected by Crohn's or colitis [3], as

\footnotetext{
* Correspondence: s.beese@bham.ac.uk

Institute of Applied Health Research, Public Health Building, University of Birmingham, Birmingham B15 2TT, UK
}

well as 2.5 million across Europe and over 1 million in the USA [4]. IBD is often diagnosed at a young age and equally in men and women. Symptoms are often debilitating and can affect day-to-day activities [5]. Patients may suffer from severe abdominal pain, bloody diarrhoea, weight loss, fatigue and other symptoms such as anaemia, vomiting and fever [6, 7]. An individual may have periods of time when their illness flares up, as well as times when they are in remission [2].

Currently, the cause of IBD is unknown; however, genetic and environmental factors are thought to play a part

(c) The Author(s). 2018 Open Access This article is distributed under the terms of the Creative Commons Attribution 4.0 International License (http://creativecommons.org/licenses/by/4.0/), which permits unrestricted use, distribution, and 
and IBD is considered as an autoimmune disease $[8,9]$. Although there is no cure, treatments include aminosalicylates and steroids to reduce inflammation and immunosuppressants to reduce the autoimmune response in the gastrointestinal tract $[10,11]$. Around $20 \%$ of patients do not respond to treatments and surgery is considered as an option to remove or repair the damaged part of the digestive system [2]. Studies have also shown that patients with IBD may have a reduced health-related quality of life compared to the general population, particularly when their disease is active and more so in women than in men $[12,13]$.

Body image is how an individual perceives themselves physically and body image dissatisfaction (BID) is associated with a distorted and negative view of oneself causing feelings of anxiousness and discomfort [14]. Generally, women are more likely to suffer with BID than men [15]. Furthermore, having negative body image can have a serious impact on health and well-being [16]. Studies have shown that patients with negative body image are more likely to suffer with depression, anxiety and suicidal feelings [17]. BID can also impact negatively upon relationships [18] and quality of life [19].

Society has an impact on an individual's perception of body image with increased pressure on both men and women to adhere to "ideal" bodies and consequentially some people are more critical of themselves [20]. Aspects such as body weight, body shape and skin problems such as acne, may contribute toward an individual's negative body image [21].

People who live with IBD may be at risk of BID for various reasons. Condition-specific symptoms like swelling of the stomach, anaemia and the less common erythema nodosum (painful red skin nodules) may be an issue $[2,22]$. Factors such as disease activity, severity and fatigue may also contribute to the dissatisfaction with body image experienced by the patient. Therapies also have an impact and steroid use is often associated with weight gain, acne, mood swings and "moon face" [23] (when fat is redistributed to the sides of the face and excess water retention occurs [24]). Surgical resection of the bowel causing cosmetic scarring and the implementation of a stoma may also add to BID [25, 26].

Most IBD patients are diagnosed at adolescence [2], a time in life when body image becomes more important and self-consciousness rises. Adolescents are at risk of mental health issues like anxiety and depression [27] and this risk is increased by long-term physical illnesses like IBD [28]. This could be further intensified by BID, leading to possible extreme reactions such as self-harm or feeling suicidal $[29,30]$.

Body image is not currently considered as an important aspect of care and treatment for IBD patients, although some research suggests that it could potentially be a problem [22]. As body image has been highlighted as an issue in other conditions such as cancer and in transplant patients [31], it is possible it may impact on IBD patients too. Studies in these areas [32, 33] have shown that body image may be associated with reduced quality of life measures.

\section{Current evidence and rationale}

Scoping searches for existing evidence were carried out in EMBASE, MEDLINE, the Cochrane Library and PROSPERO (November 2017) using various terms to encompass IBD and body image (including inflammatory bowel disease, ulcerative colitis, Crohn's disease, IBD, body image, body dissatisfaction). No existing or ongoing systematic reviews on body image in IBD were identified. However, the searches identified in excess of 20 primary studies relating to different aspects of body image in IBD. Studies variously explored measurement tools for BID, prevalence, factors associated with BID in IBD and quality-of-life. A systematic review is therefore warranted to synthesise and clarify this heterogeneous evidence base.

The following four questions will be addressed in this systematic review in order to develop an understanding of the evidence around BID in IBD patients:

1. What tools are used to measure body image in IBD patients and what are their components?

2. What is the prevalence and severity of body image dissatisfaction in IBD patients?

3. What factors are associated with body image dissatisfaction in IBD patients?

4. Is there an association between body image dissatisfaction and quality of life in IBD patients?

\section{Methods}

This systematic review protocol has been prepared according to the Preferred Reporting Items of Systematic Reviews and Meta-Analysis Protocols (PRISMA-P) [34] statement and is also registered on PROSPERO (CRD42018060999).

\section{Search strategy}

Bibliographic databases (EMBASE, MEDLINE, PsycINFO, Cochrane CENTRAL) will be searched using a sensitive search strategy aiming to identify any study reporting on body image in the context of IBD. Index and free text terms for IBD and body image will be combined. Strategies will be adapted for each database and run without date or language restrictions. No study design filters will be applied. Ongoing trials databases (Clinicaltrials.gov and EU Clinical Trial Register) will be searched for any current trials assessing body image. Reference lists for each article included in the systematic review will also be checked. Results will be managed using Endnote X7 (Clarivate Analytics) to facilitate automatic 
and manual removal of duplicate records, study screening and selection and record keeping. An example search strategy for MEDLINE is shown in Table 1.

\section{Screening and selection criteria}

All identified records will be screened by two reviewers (SB and $\mathrm{IH}$ ) independently based on the criteria listed below. Full texts will be acquired for any potentially relevant studies. Where articles cannot be obtained, authors will be contacted. Reasons for exclusion of studies will be recorded. Should potentially relevant non-English language studies be identified, part translation of relevant portions of articles will be undertaken where feasible. Selection criteria are as follows:

Question 1. What tools are used to measure body image in IBD patients and what are their components?

Study design: any primary studies reporting quantitative data will be eligible. The most likely study designs will be cross-sectional and cohort studies, but it is possible that relevant data may be reported as part of a randomised controlled trial. Qualitative research will be excluded.

Table 1 Example search strategy for MEDLINE database

\begin{tabular}{ll}
\hline Search & Query \\
\hline$\# 1$ & exp inflammatory bowel diseases/ \\
$\# 2$ & inflammatory bowel disease*.mp. \\
$\# 3$ & exp Colitis, Ulcerative/ \\
$\# 4$ & ulcerative colitis.mp. \\
$\# 5$ & exp Crohn disease/ \\
$\# 6$ & Crohn* disease.mp. \\
$\# 7$ & Crohn*.mp. \\
$\# 8$ & IBD.mp. \\
$\# 9$ & CD.mp. \\
$\# 10$ & UC.mp. \\
$\# 11$ & 1 OR 2 OR 3 OR 4 OR 5 OR 6 OR 7 OR 8 OR 9 OR 10 \\
$\# 12$ & exp body image/ \\
$\# 13$ & body image.mp. \\
$\# 14$ & body dissatisfaction.mp. \\
$\# 15$ & body awareness.mp. \\
$\# 16$ & body concern*.mp. \\
$\# 17$ & body attitude*.mp. \\
$\# 18$ & body preoccupation.mp. \\
$\# 19$ & body perception.mp. \\
$\# 20$ & body anxiety.mp. \\
$\# 21$ & body conscious*.mp. \\
$\# 23$ & 12 OR 13 OR 14 OR 15 OR 16 OR 17 OR 18 OR 19 OR 20 OR 21 \\
\hline &
\end{tabular}

Publication type: conference abstracts identified in searches of bibliographic databases will be included if they were published up to 3 years before date of searches and no subsequent full publication can be identified.

Population: patients of any age diagnosed with IBD. At least $50 \%$ of population must have IBD unless results are reported separately for sub-groups of individuals with IBD.

Tools: any tool measuring any aspect of body image (including relevant domains or questions of quality of life tools). Tools specific to certain subgroups, e.g. stoma-based questionnaires will only be included if relevant questions on body image are part of the tool.

The following eligibility criteria will additionally need to be met for studies to be relevant for each of questions $2-4$ :

Question 2. What is the prevalence of body image dissatisfaction in IBD patients?

Include: studies that report prevalence/frequency and severity of BID (including mean/median body image scores) in IBD patients.

Question 3. What factors are associated with body image dissatisfaction in IBD patients?

Include: studies where data is reported on associations between any factor in inflammatory bowel disease patients and BID. This might include factors such as age, gender, disease activity, medication, age at onset and disease subtype.

Question 4. Is there an association between body image dissatisfaction and quality of life in IBD patients?

Include: studies that have looked at the association between results from body image tools and quality of life tools in patients with a diagnosis of inflammatory bowel disease. This might include an association between two separate domains of the same tool (e.g. body image and physical quality of life).

\section{Data extraction}

Data extraction will be performed independently by two reviewers, SB and $\mathrm{IH}$, with discrepancies resolved through discussion or referral to a third reviewer (DM). A pre-designed piloted data extraction sheet will be used. Authors will be contacted if any clarification of reported information is required. Data to be extracted will include study characteristics, patient characteristics and data needed for analysis, with examples shown below.

\section{Study characteristics}

Study characteristics include study design, inclusion/exclusion criteria for study, recruitment methods, aim of study, follow-up period and study setting. 


\section{Participant characteristics}

Participant characteristics include number of patients, age, gender, type of inflammatory bowel disease, severity of disease, disease activity, BMI, comorbidities and therapy/surgery.

\section{Data for synthesis/analysis}

Data for synthesis include body image measurement tool, components of body image tools/scales, data on body image dissatisfaction (e.g. body image scores, prevalence, thresholds for determining BID), factors associated with body image dissatisfaction and how this has been quantified, quality of life measures and association between body image and quality of life.

\section{Quality assessment}

Assessment of study quality will be performed alongside data extraction. SB and $\mathrm{IH}$ will independently undertake quality assessment, with discrepancies resolved through discussion or referral to DM. Quality assessment will be based on checklists from the Joanna Briggs Institute critical appraisal tools [35]. The most relevant checklists will be those for cross-sectional analytical studies, cross-sectional prevalence studies and cohort studies. Other study designs may report relevant data; for example, relevant cross-sectional data may be presented in the context of a randomised controlled trial. In this case, the quality items for cross-sectional studies will still be relevant.

Important quality items will likely relate, for example, to sample selection, response rate during enrolment in the study and measurement of outcomes in a valid and reliable way.

\section{Synthesis/analysis}

Evidence for each of the four questions will be synthesised separately. Summary tables of study characteristics will be produced for each question as well as an overall risk of bias table. Given the anticipated heterogeneity (e.g. differences in study design, measurement tools, population and outcome statistics), it is likely that synthesis will be narrative, with key findings tabulated. The feasibility of meta-analysis will be considered but will be dependent on clinical and methodological homogeneity. If feasible, a random effects model is more likely to be appropriate.

Relevant outcome metrics that would be considered for pooling are BID prevalence proportions or mean body image scores, odds ratios/risk ratios or correlation coefficients relating to the association between different factors. If meta-analysis is carried out, the $I^{2}$ statistic will be used to measure the percentage of variation across studies that is due to heterogeneity beyond that expected by chance. If meta-analysis is not feasible, it may still be possible to present findings in forest plots (without pooling) for illustrative purposes.
Important subgroups relate to disease type and severity, body image measurement tool, gender and age. Where possible, findings will be presented according to different diseases/disease severities and different tools. Further consideration of subgroups will likely be limited to those reported within primary studies. Quality assessment findings will be used in considering the strength of evidence for each of the four questions. Formal sensitivity analysis based on quality assessment findings is unlikely to be feasible. Funnel plots will be used to assess publication bias alongside Egger's test [36] for each outcome were meta-analysis is possible and where there are ten or more studies contributing data.

\section{Discussion}

To the best of our knowledge, this will be the first systematic review exploring BID in IBD patients. It will reveal all evidence on BID in IBD including how it is measured, the prevalence and severity of BID, possible risk factors in IBD patients and any associations between BID and quality of life. Any gaps in the evidence base will also be highlighted, which will be useful for informing future research recommendations.

Whilst NICE quality standards for IBD recognise enhancing quality of life in their outcome framework [37], neither this nor any other guidelines currently consider body image factors in the treatment and care decision-making in IBD. At present, it is unclear to what extent BID issues impact on quality of life and which, if any, quality of life measures are capturing aspects relating to BID. Elucidating the relationship between body image dissatisfaction and quality of life in this patient group is therefore important.

It is known that individuals with BID are at risk of depression, anxiety and potentially even self-harm [38]. It is also known that individuals are more likely to suffer with mental health issues if they have a long-term physical health condition [39]. If BID is prevalent in IBD patients, they could be at increased risk of these conditions. Therefore, it is important that IBD clinicians and patients are made aware of any risks, which would allow for early intervention to prevent or ameliorate BID. If certain risk factors are found to be associated with BID in IBD patients, these could be used to identify which individuals are particularly at risk.

Ultimately, this might lead to interventions being developed and implemented that aim to improve body image, thereby reducing further mental health risks and improving quality of life.

\section{Abbreviations}

BID: Body image dissatisfaction; IBD: Inflammatory bowel disease;

PRISMA: Preferred Reporting Items for Systematic Reviews and MetaAnalyses; PRISMA-P: Preferred Reporting Items for Systematic Review and Meta-Analysis-Protocols 


\section{Acknowledgements}

Not applicable.

\section{Funding}

During this research, Isobel Harris was funded by a National Institute for Health Research (NIHR). Research Methods_-Systematic Review Fellowship and Sophie Beese was a locally funded trainee in systematic reviews at the University of Birmingham with agreement from the NIHR. This article presents independent research funded by the NIHR. The views expressed are those of the authors and not necessarily those of the NHS, the NIHR or the Department of Health.

\section{Availability of data and materials}

Not applicable.

\section{Authors' contributions}

SB identified the topic, undertook scoping, defined the question, developed the protocol and wrote the draft of the manuscript. $I H$ contributed to the methods and commented on and approved the final version of this paper. DM provided methodological input to aid protocol development and read, commented on and edited the draft and approved the final version. JD provided methodological input and read, commented on and edited the draft and approved the final version.

\section{Ethics approval}

Not applicable.

\section{Consent for publication}

Not applicable.

\section{Competing interests}

The authors declare that they have no competing interests.

\section{Publisher's Note}

Springer Nature remains neutral with regard to jurisdictional claims in published maps and institutional affiliations.

\section{Received: 11 April 2018 Accepted: 17 October 2018}

\section{Published online: 13 November 2018}

\section{References}

1. Malik TA. Inflammatory bowel disease: historical perspective, epidemiology, and risk factors. Surg Clin N Am. 2015;95(6):1105-22.

2. NHS Choices: Inflammatory bowel disease. http://www.nhs.uk/conditions/ Inflammatory-bowel-disease/Pages/Introduction.aspx (2015). Accessed 17 Feb 2017.

3. Crohn's and Colitis UK: about inflammatory bowel disease. https://www. crohnsandcolitis.org.uk/about-inflammatory-bowel-disease Accessed 18 Feb 2017.

4. Kaplan GG. The global burden of IBD: from 2015 to 2025. Nat Rev Gastroenterol Hepatol. 2015;12(12):720-7.

5. Mosli M, Al Beshir M, Al-Judaibi B, Al-Ameel T, Saleem A, Bessissow T, Ghosh S, Almadi M. Advances in the diagnosis and management of inflammatory bowel disease: challenges and uncertainties. Saudi J Gastroenterol. 2014; 20(2):81-101.

6. Centers for Disease Control and Prevention: what is inflammatory bowel disease (IBD)? https://www.cdc.gov/ibd/what-is-ibd.htm (2014). Accessed: 30 Jan 2017.

7. Sobczak M, Fabisiak A, Murawska N, Wesolowska E, Wierzbicka P, Wlazlowski M, Wojcikowska M, Zatorski H, Zwolinska M, Fichna J. Current overview of extrinsic and intrinsic factors in etiology and progression of inflammatory bowel diseases. Pharmacol Reports. 2014;66(5):766-75.

8. Uniken Venema WT, Voskuil MD: The genetic background of inflammatory bowel disease: from correlation to causality. J Pathol. 2017;241(2):146-58.

9. Bernstein CN. Treatment of IBD: where we are and where we are going. Am J Gastroenterol. 2015;110(1):114-26.

10. National Institute for Health and Care Excellence: ulcerative colitis: management CG166. https://www.nice.org.uk/guidance/cg166 (2013). Accessed: 15 Feb 2017
11. National Institute for Health and Care Excellence: Crohn's disease: Management CG152. https://www.nice.org.uk/guidance/cg152 (2012). Accessed: 18 Feb2017

12. Kalafateli M, Triantos C, Theocharis G, Giannakopoulou D, Koutroumpakis E, Chronis A, Sapountzis A, Margaritis V, Thomopoulos K, Nikolopoulou V. Health-related quality of life in patients with inflammatory bowel disease: a single-center experience. Annals of gastroenterology: quarterly publication of the Hellenic society of. Gastroenterology. 2013;26(3):243-8.

13. Magalhaes J, Castro FD, Carvalho PB, Moreira MJ, Cotter J. Quality of life in patients with inflammatory bowel disease: importance of clinical, demographic and psychosocial factors. Arq Gastroenterol. 2014;51(3):192-7.

14. National Eating Disorders Association: What is body image? https://www. nationaleatingdisorders.org/what-body-image Accessed: 17 Feb 2017.

15. Cash TF, Morrow JA, Hrabosky Jl, Perry AA. How has body image changed? A cross-sectional investigation of college women and men from 1983 to 2001. J Consult Clin Psychol. 2004;72(6):1081-9.

16. Griffiths S, Hay P, Mitchison D, Mond JM, McLean SA, Rodgers B, Massey R, Paxton $\mathrm{SJ}$. Sex differences in the relationships between body dissatisfaction, quality of life and psychological distress. Aust N Z J Public Health. 2016;40(6):518-22.

17. Grogan S. Body image: understanding body dissatisfaction in men, women and children. London: Taylor \& Francis; 2016.

18. Dyl J, Kittler J, Phillips KA, Hunt Jl. Body dysmorphic disorder and other clinically significant body image concerns in adolescent psychiatric inpatients: prevalence and clinical characteristics. Child Psychiatry Hum Dev. 2006;36(4):369-82

19. Kim JS, Kang S. A study on body image, sexual quality of life, depression, and quality of life in middle-aged adults. Asian Nurs Res (Korean Soc Nurs Sci). 2015;9(2):96-103.

20. Brown Z, Tiggemann M. Attractive celebrity and peer images on Instagram: effect on women's mood and body image. Body Image. 2016;19:37-43.

21. Holland G, Tiggemann M. A systematic review of the impact of the use of social networking sites on body image and disordered eating outcomes. Body Image. 2016;17:100-10.

22. McDermott E, Mullen G, Moloney J, Keegan D, Byrne K, Doherty GA, Cullen G, Malone K, Mulcahy HE. Body image dissatisfaction: clinical features, and psychosocial disability in inflammatory bowel disease. Inflamm Bowel Dis. 2015;21(2):353-60

23. NHS choices: corticosteroids - side effects. http://www.nhs.uk/Conditions/ Corticosteroid-(drugs)/Pages/Sideeffects.aspx (2015). Accessed: 19 Feb 2017.

24. Liu D, Ahmet A, Ward L, Krishnamoorthy P, Mandelcorn ED, Leigh R, Brown $J \mathrm{P}$, Cohen A, Kim H. A practical guide to the monitoring and management of the complications of systemic corticosteroid therapy. Allergy Asthma Clin Immunol. 2013:9(1):30.

25. Polle SW, Dunker MS, Slors JFM, Sprangers MA, Cuesta MA, Gouma DJ, Bemelman WA. Body image, cosmesis, quality of life, and functional outcome of hand-assisted laparoscopic versus open restorative proctocolectomy: longterm results of a randomized trial. Surg Endosc. 2007;21(8):1301-7.

26. Spinelli A, Carvello M, D'Hoore A, Pagnini F. Psychological perspectives of inflammatory bowel disease patients undergoing surgery: rightful concerns and preconceptions. Curr Drug Targets. 2014;15(11):1074-8.

27. Merikangas KR, Nakamura EF, Kessler RC. Epidemiology of mental disorders in children and adolescents. Dialogues Clin Neurosci. 2009;11(1):7-20.

28. Weiland SK, Pless IB, Roghmann KJ. Chronic illness and mental health problems in pediatric practice: results from a survey of primary care providers. Pediatrics. 1992:89(3):445-9.

29. Pinto ACS, Luna IT, Sivla AA, Pinheiro PNC, Braga VAB, AMAe S. Risk factors associated with mental health issues in adolescents: an integrative review. Revista da Escola de Enfermagem da USP. 2014;48:555-64.

30. Michaud P-A, Fombonne E. Common mental health problems. Br Med J. 2005;330(7495):835-8

31. Fingeret MC, Teo I, Epner DE. Managing body image difficulties of adult cancer patients: lessons from available research. Cancer. 2014;120(5):633-41.

32. McClelland SI, Holland KJ, Griggs JJ. Quality of life and metastatic breast cancer: the role of body image, disease site, and time since diagnosis. Qual Life Res. 2015;24(12):2939-43.

33. Yagil $Y$, Geller $S$, Sidi $Y$, Tirosh $Y$, Katz $P$, Nakache $R$. The implications of body-image dissatisfaction among kidney-transplant recipients. Psychol Health Med. 2015;20(8):955-62.

34. Moher D, Liberati A, Tetzlaff J, Altman DG, The PRISMA Group. Preferred Reporting Items for Systematic Reviews and Meta-Analyses: The PRISMA Statement. PLoS Med. 2009;6(7):e1000097. 
35. The Joanna Briggs Institute. The Joanna Briggs Institute Reviewers' Manual 2008 Edition. Adelaide: The Joanna Briggs Institute; 2008.

36. Carpenter J, Egger M, Harbord R, Higgins J, Jones D, Moher D, Sterne J, Sutton A, Tetzlaff J: Addressing reporting biases. In: Cochrane Handbook for Systematic Reviews of Intervention. Version 5.1.0 (updated March 2011) edn, Edited by Sterne JAC, Egger M, D M: The Cochrane Collaboration; 2011.

37. National Institute for Health and Care Excellence: Inflammatory Bowel Disease Quality Standard. In., vol. QS81; 2015.

38. Muehlenkamp JJ, Brausch AM. Body image as a mediator of non-suicidal self-injury in adolescents. J Adolesc. 2012;35(1):1-9.

39. Katon WJ. Clinical and health services relationships between major depression, depressive symptoms, and general medical illness. Biol Psychiatry. 2003;54(3):216-26.

Ready to submit your research? Choose BMC and benefit from:

- fast, convenient online submission

- thorough peer review by experienced researchers in your field

- rapid publication on acceptance

- support for research data, including large and complex data types

- gold Open Access which fosters wider collaboration and increased citations

- maximum visibility for your research: over $100 \mathrm{M}$ website views per year

At $\mathrm{BMC}$, research is always in progress.

Learn more biomedcentral.com/submissions 